\title{
Lie Group Analysis for the Effects of Variable Fluid Viscosity and Thermal Radiation on Free Convective Heat and Mass Transfer with Variable Stream Condition
}

\author{
P. Loganathan ${ }^{1}$, P. Puvi Arasu ${ }^{2}$ \\ ${ }^{1}$ Department of Mathematics, Anna University, Chennai, India \\ ${ }^{2}$ Erode Sengunthar Engineering College, Thudupathi, India \\ E-mail: puviarasup@gmail.com \\ Received December 23, 2009; revised February 26, 2010; accepted March 6, 2010
}

\begin{abstract}
Natural convective boundary layer flow and heat and mass transfer of a fluid with variable viscosity and thermal radiation over a vertical stretching surface in the presence of suction/injection is investigated by Lie group analysis. Fluid viscosity is assumed to vary as a linear function of temperature. The symmetry groups admitted by the corresponding boundary value problem are obtained by using a special form of Lie group transformations viz. scaling group of transformations. An exact solution is obtained for translation symmetry and numerical solutions for scaling symmetry. The effects of fluid viscosity and thermal radiation on the dimensionless velocity, temperature and concentration profiles are shown graphically. Comparisons with previously published works are performed and excellent agreement between the results is obtained. The conclusion is drawn that the flow field and temperature profiles are significantly influenced by these parameters.
\end{abstract}

Keywords: Scaling Group of Transformations, Free Convective Flow, Temperature-Dependent Fluid Viscosity, Suction/Blowing, Thermal Radiation

\section{Introduction}

The study of natural convection flow for an incompressible viscous fluid past a heated surface has attracted the interest of many researchers in view of its important applications to many engineering problems such as cooling of nuclear reactors, the boundary layer control in aerodynamics, crystal growth, food processing and cooling towers. In this paper, symmetry methods are applied to a natural convection boundary layer problem. The main advantage of such methods is that they can successfully be applied to non-linear differential equations. The symmetries of differential equations are those continuous groups of transformations under which the differential equations remain invariant, that is, a symmetry group maps any solution to another solution. The symmetry solutions are quite popular because they result in the reduction of the number of independent variables of the problem.

A class of flow problems with obvious relevance to polymer extrusion is the flow induced by the stretching motion of a flat elastic sheet. In a melt-spinning process, the extrudate from the die is generally drawn and simul- taneously stretched into a filament or sheet, which is thereafter solidified through rapid quenching or gradual cooling by direct contact with water or chilled metal rolls. In fact, stretching imports a unidirectional orientation to the extrudate, thereby improving its mechanical properties and the quality of the final product greatly depends on the rate of cooling. Crane [1] was the first who studied the motion set up in the ambient fluid due to a linearly stretching surface. Several authors see e.g., the references cited in [2], have subsequently explored various aspects of the accompanying heat transfer occurring in the infinite fluid medium surrounding the stretching sheet. The hydrodynamics of a finite fluid medium, i.e. a thin liquid film, on a stretching sheet was first considered by Wang [3] who by means of a similarity transformation reduced the unsteady Navier-Stokes equations to a non-linear ordinary differential equation. The accompanying heat transfer problem was solved more recently by Andersson et al. [2]. In these studies the film surface was planar and free of any stresses.

The production of sheeting material arises in a number of industrial manufacturing processes and includes both metal and polymer sheets. It is well known that the flow 
in a boundary layer separates in the regions of adverse pressure gradient and the occurrence of separation has several undesirable effects in so far as it leads to increase in the drag on the body immersed in the flow and adversely affects the heat transfer from the surface of the body. Several methods have been developed for the purpose of artificial control of flow separation. Separation can be prevented by suction as the low-energy fluid in the boundary layer is removed [4,5]. On the contrary, the wall shear stress and hence the friction drag is reduced by blowing. Free convective phenomenon has been the object of extensive research. The importance of this phenomenon is increasing day by day due to the enhanced concern in science and technology about buoyancy induced motions in the atmosphere, the bodies in water and quasisolid bodies such as earth. Natural convection flows driven by temperature differences are very much interesting in case of Industrial applications. Buoyancy plays an important role where the temperature differences between land and air give rise to a complicated flow and in enclosures such as ventilated and heated rooms (Elbashbeshy and Bazid [6]).

So such type of problem, which we are dealing with, is very much useful to polymer technology and metallurgy. Cheng and Minkowycz [7] and Cheng [8] studied the free convective flow in a saturated porous medium. Wilks [9] had studied the combined forced and free convection flow along a semi-infinite plate extending vertically upwards with its leading edge horizontal. Boutros et al. [10] solved the steady free convective boundary layer flow on a nonisothermal vertical plate. Recently, any studies were made on the steady free convective boundary layer flow on moving vertical plates considering the effect of buoyancy forces on the boundary layer Chen and Strobel [11], Ramachandran et al. [12], Lee and Tsai [13]. The radiative effects have important applications in physics and engineering particularly in space technology and high temperature processes. But very little is known about the effects of radiation on the boundary layer. Thermal radiation effects may play an important role in controlling heat transfer in polymer processing industry where the quality of the final product depends on the heat controlling factors to some extent. High temperature plasmas, cooling of nuclear reactors, liquid metal fluids, power generation systems are some important applications of radiative heat transfer from a vertical wall to conductive gray fluids. The effect of radiation on heat transfer problems have studied by Hossain and Takhar [14], Takhar et al. [15], Hossain et al. [16]. In all of the above mentioned studies, fluid viscosity was assumed to be constant. However, it is known that the physical properties of fluid may change significantly with temperature. For lubricating fluids, heat generated by the internal friction and the corresponding rise in temperature affects the viscosity of the fluid and so the fluid viscosity can no longer be assumed constant. The increase of temperature leads to a local increase in the transport phenomena by reducing the viscosity across the momentum boundary layer and so the heat transfer rate at the wall is also affected. Therefore, to predict the flow behaviour accurately it is necessary to take into account the viscosity variation for incompressible fluids. Gary et al. [17] and Mehta and Sood [18] showed that, when this effect is included the flow characteristics may changed substantially compared to the constant viscosity assumption. Mukhopadhyay et al. [19] investigated the MHD boundary layer flow with variable fluid viscosity over a heated stretching sheet. Recently, Mukhopadhyay and Layek [20] studied the effects of thermal radiation and variable fluid viscosity on free convective flow and heat transfer past a porous stretching surface.

Many authors have constructed an exponential type of exact solution using the translation symmetry and a series type of approximate solution using the scaling symmetry and also discussed some boundary value problems. So far no attempt has been made to study the heat and mass transfer in a vertical stretching surface using Lie groups and hence we study the problem of natural convection heat and mass transfer flow past a stretching sheet for various parameters using Lie group analysis.

\section{Mathematical Analysis}

We consider a free convective, laminar boundary layer flow and heat and mass transfer of viscous incompressible fluid over a vertical stretching sheet emerging out of a slit at origin $(x=0, y=0)$ and moving with non-uniform velocity $U(x)$ in the presence of thermal radiation (Figure 1).

The governing equations of such type of flow are, in the usual notations,

$$
\frac{\partial u}{\partial x}+\frac{\partial v}{\partial y}=0
$$

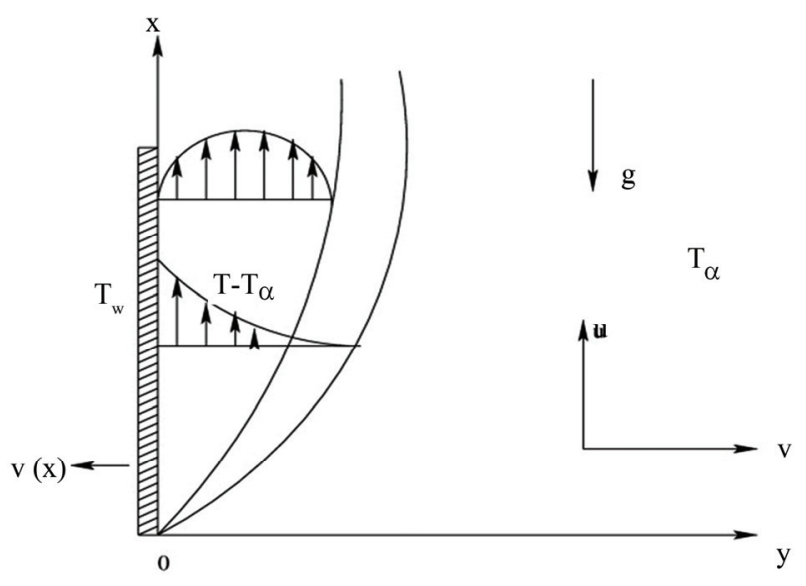

Figure 1. Physical model of boundary layer flow over a vertical stretching surface. 


$$
\begin{gathered}
u \frac{\partial u}{\partial x}+v \frac{\partial u}{\partial y}= \\
\frac{1}{\rho} \frac{\partial \mu}{\partial T} \frac{\partial T}{\partial y} \frac{\partial u}{\partial y}+\frac{\mu}{\rho} \frac{\partial^{2} u}{\partial x^{2}}+\left[g \beta\left(T-T_{\infty}\right)+g \beta^{*}\left(C-C_{\infty}\right)\right] \\
u \frac{\partial T}{\partial x}+v \frac{\partial T}{\partial y}=\frac{\kappa}{\rho c_{p}} \frac{\partial^{2} T}{\partial y^{2}}-\frac{1}{\rho c_{p}} \frac{\partial q_{r}}{\partial y} \\
u \frac{\partial C}{\partial x}+v \frac{\partial C}{\partial y}=D \frac{\partial^{2} C}{\partial y^{2}} \\
u=U(x), v=-V(x), C=C_{w}, T=T_{w} \text { at } y=0 \\
u=0, C=C_{\infty}, T=T_{\infty} \text { as } y \rightarrow \infty
\end{gathered}
$$

when the viscous dissipation term in the energy equation is neglected (as the fluid velocity is very low). Here $u$ and $v$ are the components of velocity respectively in the $x$ and $y$ directions, $\mu$ is the coefficient of fluid viscosity, $\rho$ is the fluid density (assumed constant), $T$ is the temperature, $\kappa$ is the thermal conductivity of the fluid, $D$ is diffusional coefficient, $\beta$ is the volumetric coefficient of thermal expansion, $\beta^{*}$ is the volumetric coefficient of concentration expansion, $\mathrm{g}$ is the gravity field, $T_{\infty}$ is the temperature at infinity, where $U(x)$ is the stream wise velocity and $V(x)$ is the velocity of suction of the fluid, $T_{w}$ is the wall temperature.

Using Rosseland approximation for radiation (Brewster [21]) we can write $q_{r}=-\frac{4 \sigma_{1}}{3 k^{*}} \frac{\partial T^{4}}{\partial y}$ where $\sigma_{1}$ is the Stefan-Boltzman constant, $k^{*}$ is the absorption coefficient.

Assuming that the temperature difference within the flow is such that $T^{4}$ may be expanded in a Taylor series and expanding $T^{4}$ about $T_{\infty}$ and neglecting higher orders we get $T^{4} \cong 4 T_{\infty}^{3} T-3 T_{\infty}{ }^{4}$

Therefore, the Equation (3) becomes

$$
u \frac{\partial T}{\partial x}+v \frac{\partial T}{\partial y}=\frac{\kappa}{\rho c_{p}} \frac{\partial^{2} T}{\partial y^{2}}-\frac{16 \sigma_{1} T_{\infty}^{3}}{3 \rho c_{p} k^{*}} \frac{\partial^{2} T}{\partial y^{2}}
$$

We now introduce the following relations for $u, v, \theta$ and $\varphi$ as

$$
u=\frac{\partial \psi}{\partial y}, v=-\frac{\partial \psi}{\partial x}, \theta=\frac{T-T \infty}{T_{w}-T_{\infty}} \text { and } \varphi=\frac{C-C_{\infty}}{C_{w}-C_{\infty}}
$$

where $u$ is the stream function. The stream wise velocity and the suction/injection velocity are taken as

$$
U(x)=c x^{m}, V(x)=V_{0} x^{\frac{m-1}{2}}
$$

Here $c>0$ is constant, $T_{w}$ is the wall temperature, the power-law exponent $m$ is also constant. In this study we take $c=1$.
The temperature-dependent fluid viscosity is given by (Batchelor [22]),

$$
\mu=\mu^{*}\left[a+b\left(T_{w}-T\right)\right]
$$

where $\mu^{*}$ is the constant value of the coefficient of viscosity far away from the sheet and $a, b$ are constants and $b(>0)$. For a viscous fluid, Ling and Dybbs [23] suggest a viscosity dependence on temperature $T$ of the form $\mu=\frac{\mu_{\infty}}{\left[1+\gamma\left(T-T_{\infty}\right)\right]}$ where $c$ is a thermal property of the fluid and $\mu_{\infty}$ is the viscosity away from the hot sheet. This relation does not differ at all with our formulation. The range of temperature, i.e., $\left(T_{w}-T_{\infty}\right)$ studied here is $\left(0-23^{0}\right) C$.

Using the relations (5) in the boundary layer Equation (2) and in the energy and diffusion Equations (3) and (4) we get the following equations

$$
\begin{gathered}
\frac{\partial \psi}{\partial y} \frac{\partial^{2} \psi}{\partial x \partial y}-\frac{\partial \psi}{\partial x} \frac{\partial^{2} \psi}{\partial y^{2}}= \\
-\zeta v^{*} \frac{\partial \theta}{\partial y} \frac{\partial^{2} \psi}{\partial y^{2}}+v^{*}[a+\zeta(1-\theta)] \frac{\partial^{3} \psi}{\partial y^{3}}+g \frac{\zeta}{b}\left(\beta \theta+\beta^{*} \varphi\right) \\
\frac{\partial \psi}{\partial y} \frac{\partial \theta}{\partial x}-\frac{\partial \psi}{\partial x} \frac{\partial \theta}{\partial y}=\left(\frac{\kappa}{\rho c_{p}}+\frac{16 \sigma_{1} T_{\infty}^{3}}{3 \rho c_{p} k^{*}}\right) \frac{\partial^{2} \theta}{\partial y^{2}} \\
\frac{\partial \psi}{\partial y} \frac{\partial \varphi}{\partial x}-\frac{\partial \psi}{\partial x} \frac{\partial \varphi}{\partial y}=D \frac{\partial^{2} \varphi}{\partial y^{2}}
\end{gathered}
$$

where $\zeta=b\left(T_{w}-T_{\infty}\right), v^{*}=\frac{\mu^{*}}{\rho}$

The boundary conditions Equation (5) become

$$
\begin{aligned}
& \frac{\partial \psi}{\partial y}=x^{m}, \frac{\partial \psi}{\partial x}=V_{0} x^{\frac{m-1}{2}}, \\
& \theta=\varphi=1 \text { at } y=0 ; \frac{\partial \psi}{\partial y} \rightarrow 0, \theta \rightarrow 0, \varphi \rightarrow 0 \text { as } y \rightarrow \infty
\end{aligned}
$$

We now introduce the simplified form of Lie-group transformations namely, the scaling group of transformations (Mukhopadhyay et al. [19]),

$$
\begin{aligned}
& \Gamma: x^{*}=x e^{\varepsilon \alpha_{1}}, y^{*}=y e^{\varepsilon \alpha_{2}}, \psi^{*}=\psi e^{\varepsilon \alpha_{3}}, \\
& u^{*}=u e^{\varepsilon \alpha_{4}}, v^{*}=v e^{\varepsilon \alpha_{5}}, \theta^{*}=\theta e^{\varepsilon \alpha_{6}}, \varphi^{*}=\varphi e^{\varepsilon \alpha_{7}}
\end{aligned}
$$

Equation (13) may be considered as a point-transformation which transforms co-ordinates $(x, y, \psi, u, v, \theta, \varphi)$ to the coordinates $\left(x^{*}, y^{*}, \psi^{*}, u^{*}, v^{*}, \theta^{*}, \varphi^{*}\right)$.

Substituting (13) in (9), (10) and (11) we get, 


$$
\begin{gathered}
e^{\varepsilon\left(\alpha_{1}+2 \alpha_{2}-2 \alpha_{3}\right)}\left(\frac{\partial \psi^{*}}{\partial y^{*}} \frac{\partial^{2} \psi^{*}}{\partial x^{*} \partial y^{*}}-\frac{\partial \psi^{*}}{\partial x^{*}} \frac{\partial^{2} \psi^{*}}{\partial y^{* 2}}\right)= \\
-\zeta v^{*} e^{\varepsilon\left(3 \alpha_{2}-\alpha_{3}-\alpha_{6}\right)}\left(\frac{\partial \theta^{*}}{\partial y^{*}} \frac{\partial^{2} \psi^{*}}{\partial y^{* 2}}\right)+ \\
v^{*}[a+\zeta] e^{\varepsilon\left(3 \alpha_{2}-\alpha_{3}\right)} \frac{\partial^{3} \psi^{*}}{\partial y^{* 3}}-\zeta v^{*} e^{\varepsilon\left(3 \alpha_{2}-\alpha_{3}-\alpha_{6}\right)} \theta^{*} \frac{\partial^{3} \psi^{*}}{\partial y^{* 3}}+ \\
g \frac{\zeta}{b}\left(\beta e^{-\varepsilon \alpha_{6}} \theta^{*}+\beta^{*} e^{-\varepsilon \alpha_{7}} \varphi^{*}\right) \\
e^{\varepsilon\left(\alpha_{1}+\alpha_{2}-\alpha_{3}-\alpha_{6}\right)}\left(\frac{\partial \psi^{*}}{\partial y^{*}} \frac{\partial \theta^{*}}{\partial x^{*}}-\frac{\partial \psi^{*}}{\partial x^{*}} \frac{\partial \theta^{*}}{\partial y^{*}}\right)= \\
\left(\frac{\kappa}{\rho c_{p}}+\frac{16 \sigma_{1} T_{\infty}^{3}}{3 \rho c_{p} k^{*}}\right) e^{\varepsilon\left(2 \alpha_{2}-\alpha_{6}\right)} \frac{\partial^{2} \theta^{*}}{\partial y^{* 2}} \\
e^{\varepsilon\left(\alpha_{1}+\alpha_{2}-\alpha_{3}-\alpha_{7}\right)}\left(\frac{\partial \psi^{*}}{\partial y^{*}} \frac{\partial \varphi^{*}}{\partial x^{*}}-\frac{\partial \psi^{*}}{\partial x^{*}} \frac{\partial \varphi^{*}}{\partial y^{*}}\right)= \\
D e^{\varepsilon\left(2 \alpha_{2}-\alpha_{7}\right)} \frac{\partial^{2} \varphi^{*}}{\partial y^{* 2}}
\end{gathered}
$$

The system will remain invariant under the group of transformations $\Gamma$, we would have the following relations among the parameters, namely

$$
\begin{aligned}
& \alpha_{1}+2 \alpha_{2}-2 \alpha_{3}=3 \alpha_{2}-\alpha_{3}-\alpha_{6}=3 \alpha_{2}-\alpha_{3}= \\
& -\alpha_{6}=-\alpha_{7} ; \alpha_{1}+\alpha_{2}-\alpha_{3}-\alpha_{6}= \\
& 2 \alpha_{2}-\alpha_{6} \text { and } \alpha_{1}+\alpha_{2}-\alpha_{3}-\alpha_{7}=2 \alpha_{2}-\alpha_{7}
\end{aligned}
$$

These relations give $\alpha_{6}=\alpha_{7}=0, \alpha_{2}=\frac{1}{4} \alpha_{1}=\frac{1}{3} \alpha_{3}$.

The boundary conditions yield $\alpha_{4}=m \alpha_{1}=\frac{1}{2} \alpha_{1}$,

$\alpha_{5}=\frac{m-1}{2} \alpha_{1}=-\frac{1}{4} \alpha_{1}\left(\right.$ as $\left.m=\frac{1}{2}\right)$

In view of these, the boundary conditions become

$$
\begin{aligned}
& \frac{\partial \psi^{*}}{\partial y^{*}}=x^{* \frac{1}{2}}, \frac{\partial \psi^{*}}{\partial x}=V_{0} x^{*\left(-\frac{1}{4}\right)}, \theta^{*}=\varphi^{*}=1 \text { at } y^{*}= \\
& 0 \text { and } \frac{\partial \psi^{*}}{\partial y^{*}} \rightarrow 0, \theta^{*} \rightarrow 0, \varphi^{*} \rightarrow 0 \text { as } y^{*} \rightarrow \infty
\end{aligned}
$$

The set of transformations $\Gamma$ reduces to

$$
\begin{aligned}
& x^{*}=x e^{\varepsilon \alpha_{1}}, y^{*}=y e^{\varepsilon \frac{\alpha_{1}}{4}}, \psi^{*}=\psi e^{\varepsilon \frac{3 \alpha_{1}}{4}}, \\
& u^{*}=u e^{\varepsilon \frac{\alpha_{1}}{2}}, v^{*}=v e^{-\varepsilon \frac{\alpha_{1}}{4}}, \theta^{*}=\theta,, \varphi^{*}=\varphi
\end{aligned}
$$

Expanding by Taylor's method in powers of $\varepsilon$ and keeping terms up to the order $\varepsilon$ we get

$$
\begin{aligned}
& x^{*}-x=x \varepsilon \alpha_{1}, y^{*}-y=y \varepsilon \frac{\alpha_{1}}{4}, \psi^{*}-\psi=\psi \varepsilon \frac{3 \alpha_{1}}{4}, \\
& u^{*}-u=u \varepsilon \frac{\alpha_{1}}{2}, v^{*}-v=-v \varepsilon \frac{\alpha_{1}}{4}, \theta^{*}-\theta=\varphi^{*}-\varphi=0
\end{aligned}
$$

In terms of differentials these yield

$$
\frac{d x}{x \alpha_{1}}=\frac{d y}{y \frac{\alpha_{1}}{4}}=\frac{d \psi}{\psi \frac{3 \alpha_{1}}{4}}=\frac{d u}{u \frac{\alpha_{1}}{2}}=\frac{d v}{-v \frac{\alpha_{1}}{4}}=\frac{d \theta}{0}=\frac{d \varphi}{0}
$$

Solving the above equations we get,

$y^{*} x^{*^{\frac{-1}{4}}}=\eta, \quad \psi^{*}=x^{*^{\frac{3}{4}}} F(\eta), \theta^{*}=\theta(\eta), \varphi^{*}=\varphi(\eta)$

With the help of these relations, the (14), (15) and (16) become

$$
\begin{gathered}
2 F^{\prime 2}-3 F F^{\prime \prime}=-4 \zeta v^{*} \vartheta^{\prime} F^{\prime \prime}+ \\
4(a+\zeta) v^{*} F^{\prime \prime \prime}-4 \zeta v^{*} \theta F^{\prime \prime \prime}+4 g \frac{\zeta}{b}\left(\beta \theta+\beta^{*} \varphi\right) \\
4\left(\frac{\kappa}{\rho c_{p}}+\frac{16 \sigma_{1} T_{\infty}^{3}}{3 \rho c_{p} k^{*}}\right) \theta^{\prime \prime}+3 F \theta^{\prime}=0 \\
4 D \varphi^{\prime \prime}+3 F \varphi^{\prime}=0
\end{gathered}
$$

The boundary conditions take the following form

$$
\begin{aligned}
& F^{\prime}=1, F=\frac{4 V_{0}}{3}, \theta=\varphi=1 \text { at } \eta= \\
& 0 \text { and } F^{\prime} \rightarrow 0, \theta \rightarrow 0, \varphi \rightarrow 0 \text { as } \eta \rightarrow \infty
\end{aligned}
$$

Again, we introduce the following transformations for $\eta, F, \theta$ and $\varphi$ in Equations (19), (20) and (21):

$\eta=\left(\frac{g \beta_{1}}{b}\right)^{\alpha_{1}} v^{* b_{1}} \eta^{*}, F=\left(\frac{g \beta_{1}}{b}\right)^{\alpha_{1}^{\prime}} v^{* b_{1}^{\prime}} F^{*}$,

$\theta=\left(\frac{g \beta_{1}}{b}\right)^{\alpha_{1}^{\prime \prime}} v^{* b_{1}^{\prime \prime}} \theta^{*}, \varphi=\left(\frac{g \beta_{1}}{b}\right)^{\alpha_{1}^{\prime \prime}} v^{* b_{1}^{\prime \prime}} \varphi^{*}$ where $\beta_{1}=\frac{\beta+\beta^{*}}{2}$

Taking $F^{*}=f, \bar{\theta}=\theta$ and $\bar{\varphi}=\varphi$ the Equations (19), (20) and (21) finally take the following form

$$
\begin{gathered}
4(a+\zeta) F^{\prime \prime \prime}-4 \zeta \theta F^{\prime \prime \prime}-4 \zeta \vartheta^{\prime} F^{\prime \prime}- \\
2 F^{\prime 2}+3 F F^{\prime \prime}+4 \zeta(\theta+\varphi) \\
\frac{4}{\operatorname{Pr}}\left(1+\frac{4}{3 N}\right) \theta^{\prime \prime}+3 F \theta^{\prime}=0 \\
\frac{4}{S c} \varphi^{\prime \prime}+3 F \varphi^{\prime}=0
\end{gathered}
$$

where $\operatorname{Pr}=\frac{v^{*} \rho c_{p}}{\kappa}=\frac{\mu^{*} c_{p}}{\kappa}$ is the Prandtl number, $N=\frac{\kappa k^{*}}{4 \sigma_{1} T_{\infty}^{3}}$ is the Radiation parameter, $S c=\frac{v^{*}}{D}$ is the Schmidt number. The boundary conditions take the following forms.

$$
\begin{aligned}
& f^{\prime}=1, f=S, \theta=\varphi=1, \\
& \text { at } \eta^{*}=0 \text { and } f^{\prime} \rightarrow 0, \theta \rightarrow 0, \varphi \rightarrow 0 \text { as } \eta^{*} \rightarrow \infty
\end{aligned}
$$


where $S=\frac{4}{3} V_{0}\left(\frac{g \beta_{1}}{b}\right)^{\frac{-1}{4}} v^{\frac{-1}{2}}, S>0$ corresponds to suction and $S<0$ corresponds to injection.

\section{Numerical Solution}

The set of non-linear ordinary differential Equations (24) to (26) with boundary conditions (27) have been solved by using the R. K. Gill method, (Gill [24]) along with Shooting Technique with $\zeta, \operatorname{Pr}, S c, a$ and $N$ as prescribed parameters. The numerical solution was done using Matlab computational software. A step size of $\Delta \eta=0.001$ was selected to be satisfactory for a convergence criterion of $10^{-7}$ in nearly all cases. The value of $\eta_{\infty}$ was found to each iteration loop by assignment statement $\eta_{\infty}=\eta_{\infty}+\Delta \eta$. The maximum value of $\eta_{\infty}$, to each group of parameters $\zeta, \operatorname{Pr}, S c$, $a$ and $N$, determined when the values of unknown boundary conditions at $\eta=0$ not change to successful loop with error less than $10^{-7}$. Effects of heat and mass transfer are studied for different values of temperature-dependent viscosity at the wall of the surface and the strength of thermal radiation. In the following section, the results are discussed in detail.

\section{Results and Discussion}

To analyze the results, numerical computation has been carried out using the method described in the previous section for various values of the temperature-dependent viscosity parameter $\zeta$, suction/injection parameter $S$,

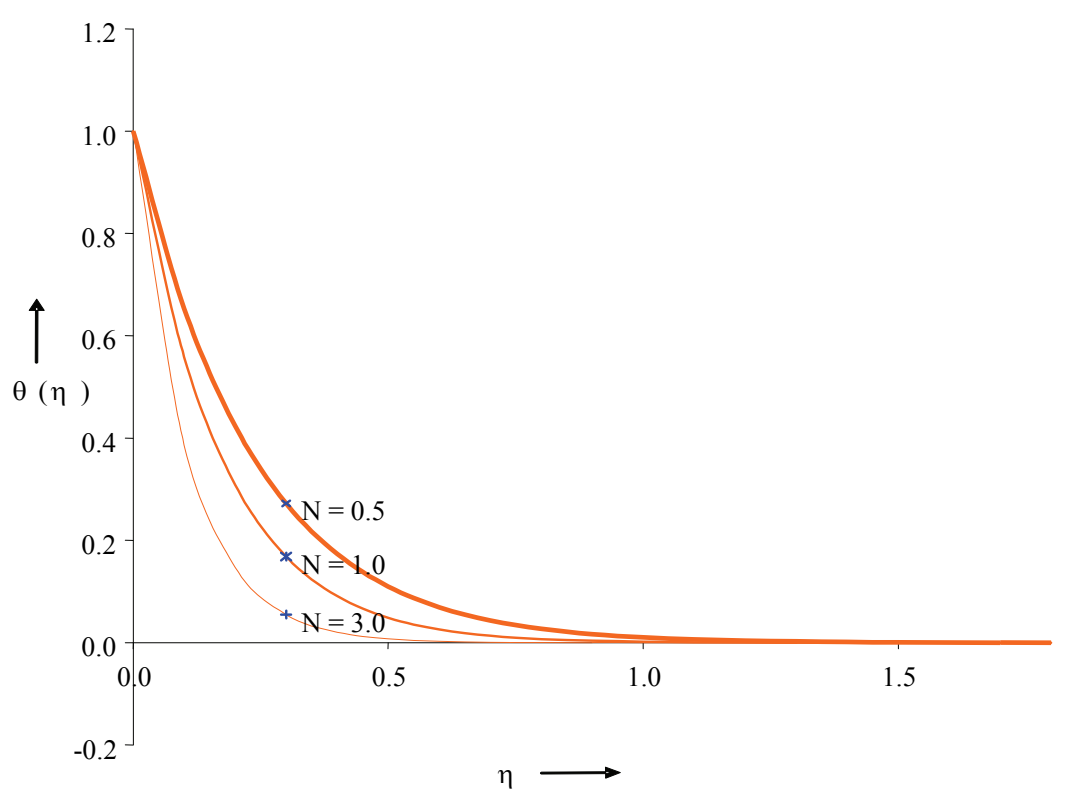

Figure 3. Effects of thermal radiation over the temperature profiles. $\operatorname{Pr}=0.3, a=1.0, S=0.5, \zeta=0.5, S c=0.62$.
Prandtl number Pr, Schmidt number Sc and radiationparameter $N$. For illustrations of the results, numericalvalues are plotted in the Figures 2-9. In all cases we take $a=1.0$.

In the absence of diffusion equations, in order to ascertain the accuracy of our numerical results, the present study is compared with the available exact solution in the literature. The temperature profiles for Prandtl number $\operatorname{Pr}$ are compared with the available exact solution of

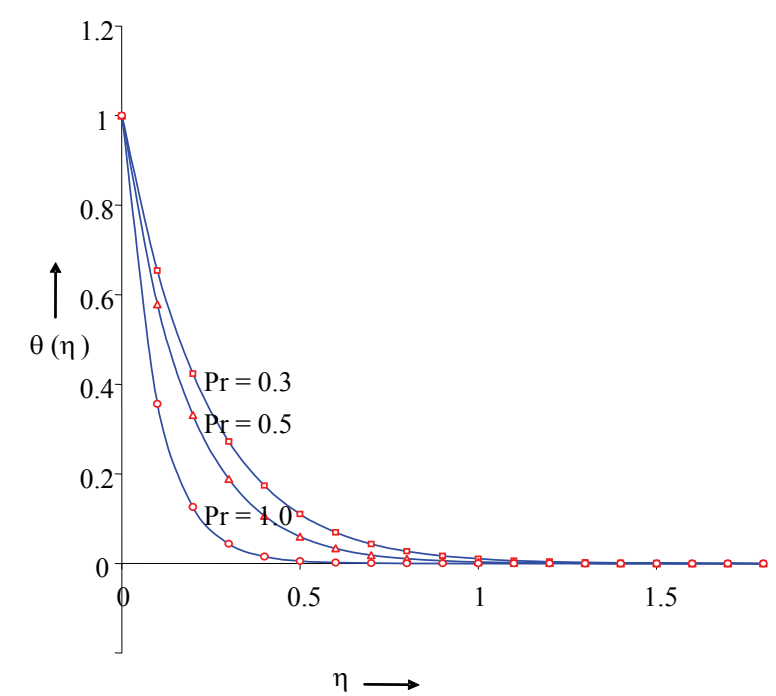

Figure 2. influence of Prandtl number over the temperature profiles. $S c=0.0, N=0.1, a=1.0, S=0.5, \zeta=0.5$.

Comparison of the temperature profiles (present result) with Mukhopadhyay and Layek [20]

Symbol: Re sult for Mukhopadhyay and Layek [20];

Solid line: Current result. 


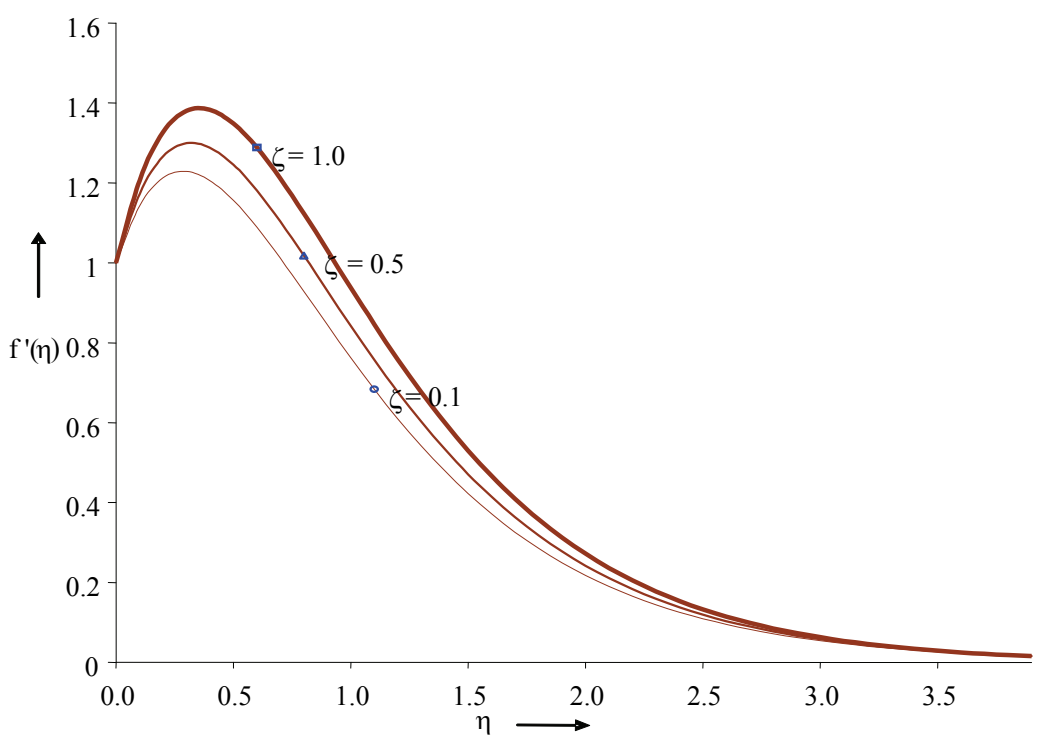

Figure 4. Effects of fluid viscosity over the velocity profiles. $S c=0.62, N=0.1, a=1.0, S=0.5, \operatorname{Pr}=0.71$.

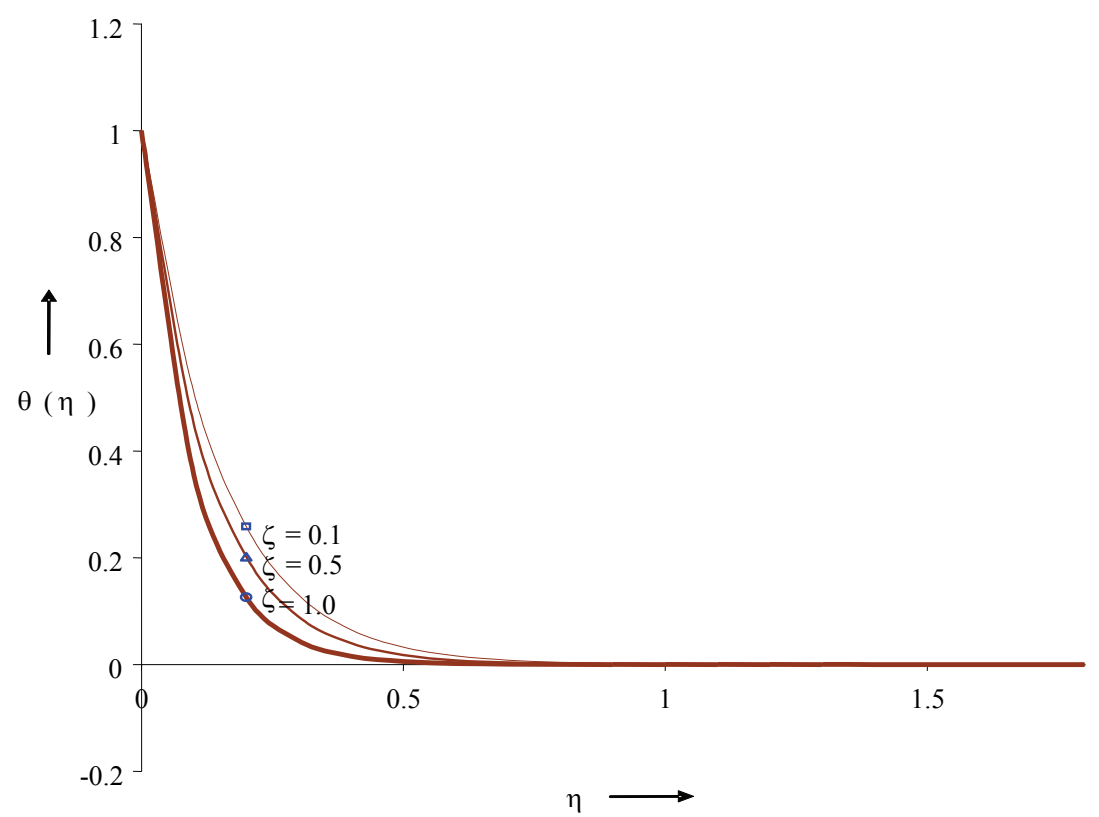

Figure 5. Effects of fluid viscosity over the temperature profiles. $S c=0.62, N=0.1, a=1.0, S=0.5, \operatorname{Pr}=0.71$.

Mukhopadhyay and Layek [20], is shown in Figure 2. It is observed that the agreements with the theoretical solution of temperature profiles are excellent. For a given $N$, it is clear that there is a fall in temperature with increaseing the Prandtl number. This is due to the fact that there would be a decrease of thermal boundary layer thickness with the increase of Prandtl number as one can see fromFigure 3 by comparing the curves with $\operatorname{Pr}=0.3$ and $\operatorname{Pr}=1.0$. This behavior implies that fluids having a smaller Prandtl number are much more responsive to thermal radiation than fluids having a larger Prandtl number.
Figure 3 illustrates the typical temperature profiles for various values of the thermal radiation parameter $N$. At a particular value of $N$, the temperature decreases with accompanying decreases in the thermal boundary layer thickness by increasing the values of Pr. Further, it is obvious that for a given $\operatorname{Pr}$, the temperature is decreased with an increase in $N$. This result can be explained by the fact that a decrease in the values of $\left(N=\frac{\kappa k^{*}}{4 \sigma_{1} T_{\infty}^{3}}\right)$ for given $k^{*}$ and $T_{\infty}$ means a decrease in the Rosseland radiation absorptivity. According to Equations (2) and (3) 


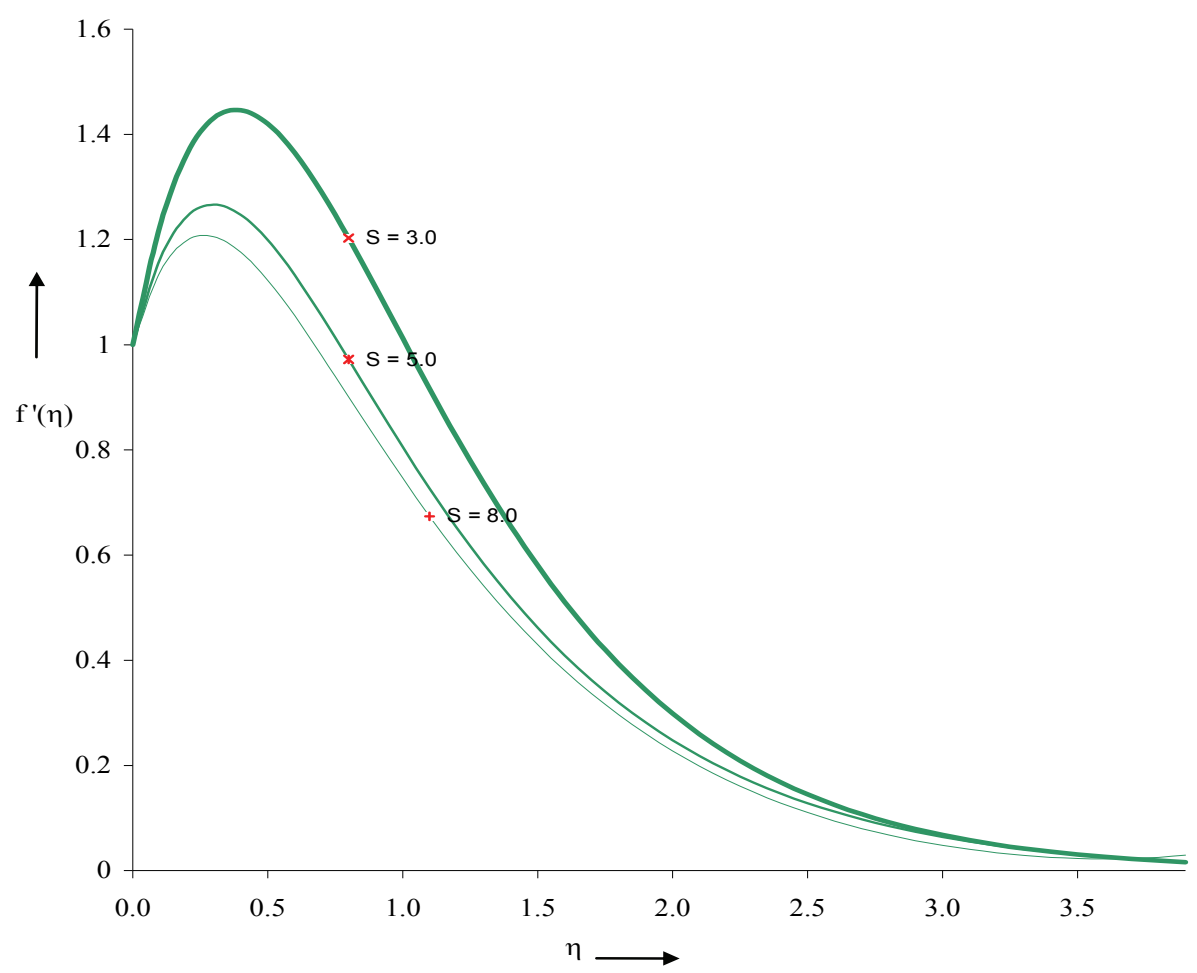

Figure 6. Effect of suction over the velocity profiles. $S=1.0, N=0.1, a=1.0, \zeta=0.5, \operatorname{Pr}=0.71$.

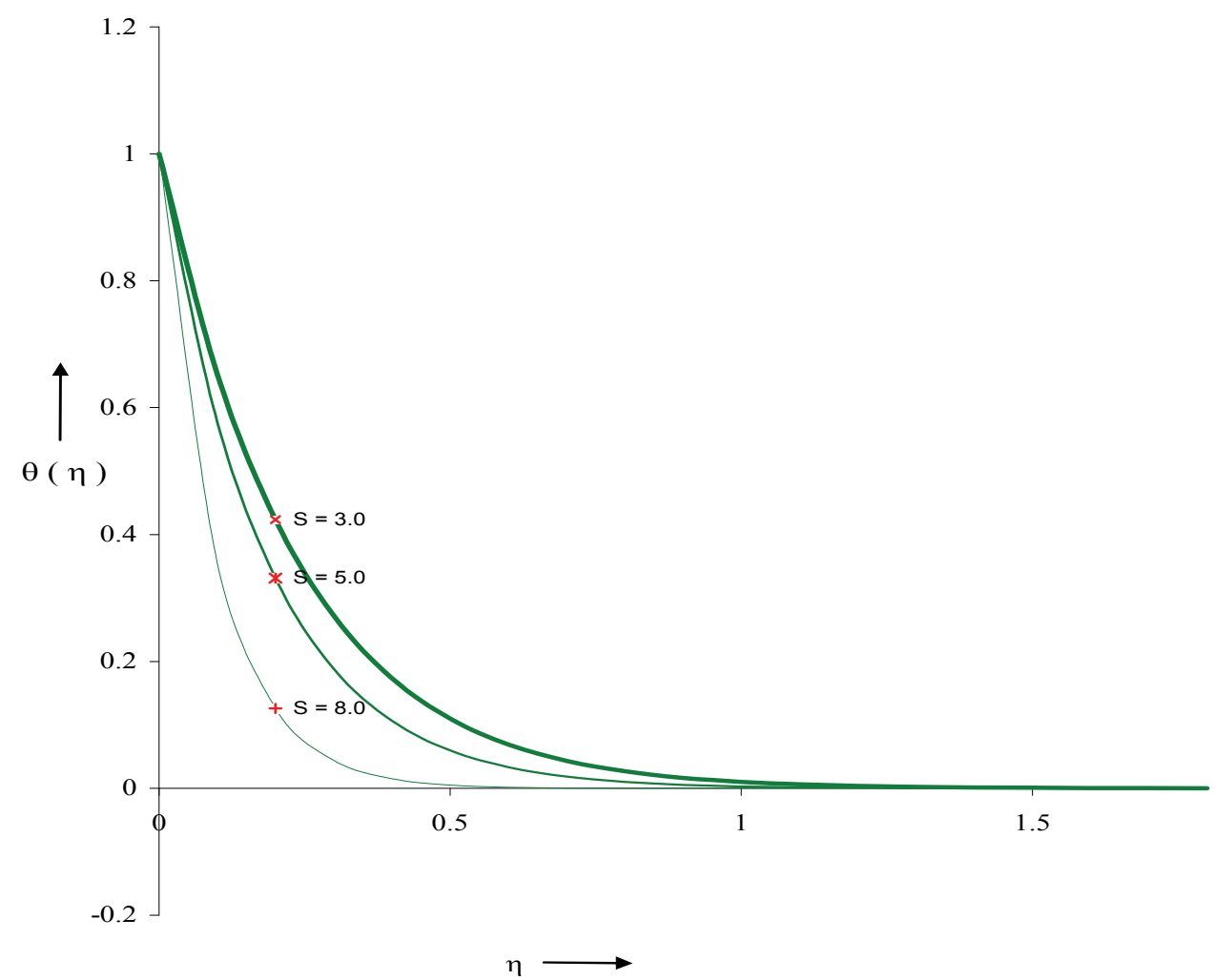

Figure 7. Influence of suction over the temperature profiles. $S c=0.62, N=0.1, a=1.0, \zeta=0.5, \operatorname{Pr}=0.71$. 


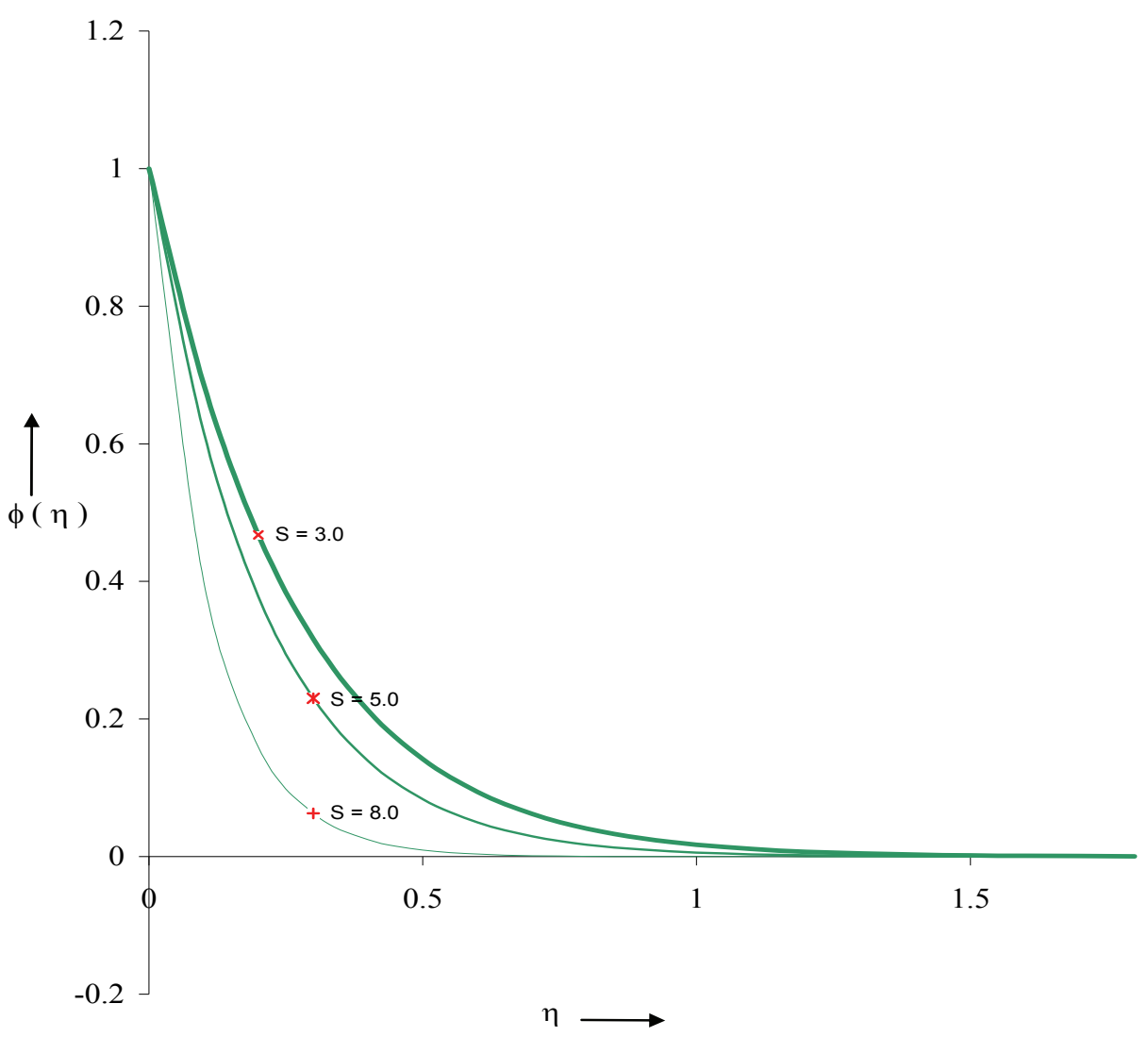

Figure 8. Effects of Suction over the concentration profiles. $S c=0.62, N=0.1, a=1.0, \zeta=0.5, \operatorname{Pr}=0.71$.

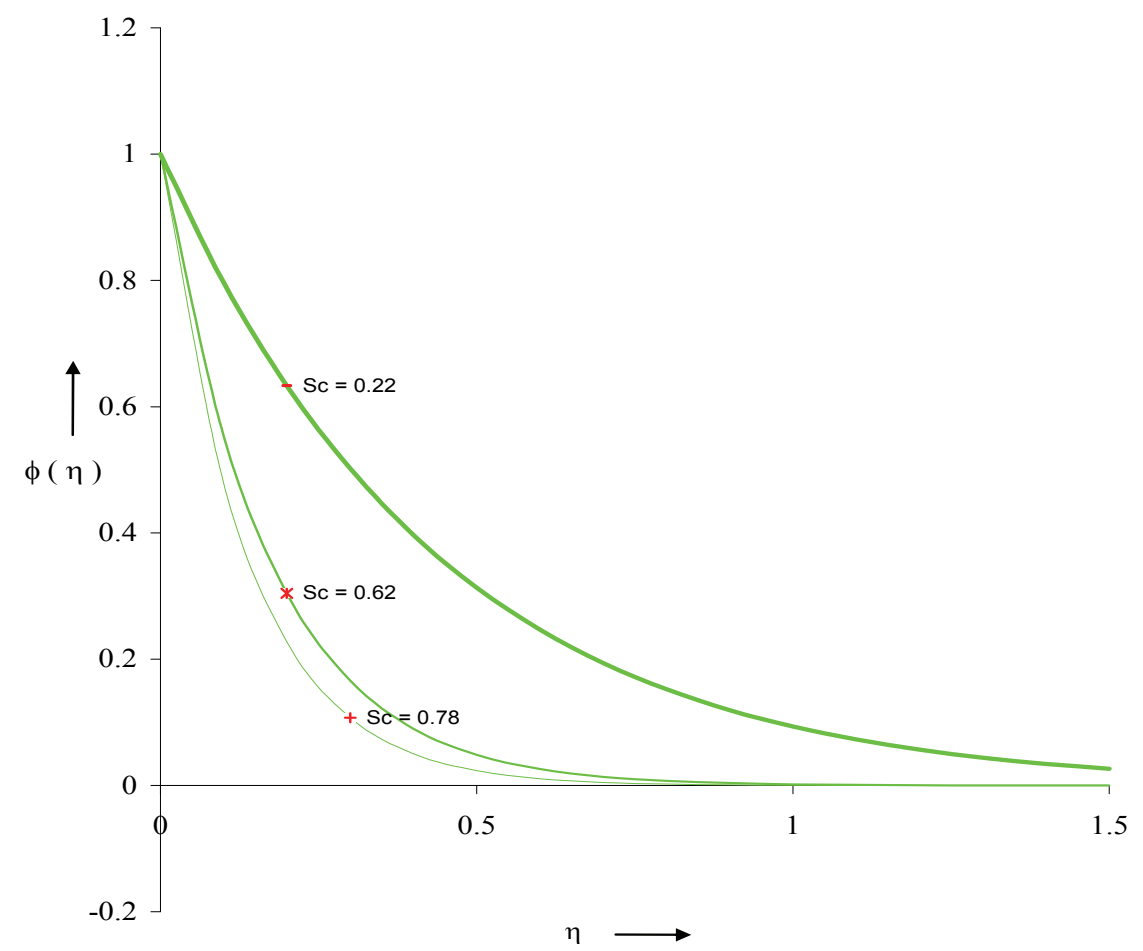

Figure 9. Effects of Schmidt number over the concentration profiles. $S=1.0, N=0.1, a=1.0, \zeta=0.5, \operatorname{Pr}=0.71$. 
the divergence of the radiative heat flux $\frac{\partial q_{r}}{\partial y}$ increases as $\kappa$ decreases which in turn increases the rate of radiative heat transferred to the fluid and hence the fluid temperature increases. In view of this explanation, the effect of radiation becomes more significant as $N \rightarrow 0(N \neq$ 0 ) and can be neglected when $N \rightarrow \infty$. Also, it is seen from Figure 3 that the larger the $N$, the thinner the thermal boundary layer thickness for both values of Pr. In addition, radiation demonstrates a more pronounced influence on the temperature distribution of $(\operatorname{Pr}=0.3)$ than that of $(\mathrm{Pr}=1.0)$.It is noticed from the figure that the temperature decreases with the increasing value of the radiation parameter $N$. The effect of radiation parameter $N$ is to reduce the temperature significantly in the flow region. The increase in radiation parameter means the release of heat energy from the flow region and so the fluid temperature decreases as the thermal boundary layer thickness becomes thinner.

Figure 4 exhibits the velocity profiles for several values of $\zeta$ with $\operatorname{Pr}=0.71$ in presence of suction $(\mathrm{S}=0.5)$ when $N=0.1$. In the case of uniform suction, the velocity of the fluid is found to increase with the increase of the temperature-dependent fluid viscosity parameter $\zeta$ at a particular value of $\eta$ except very near the wall as well as far away of the wall (at $\eta=5$ ). This means that the velocity decreases (with the increasing value of $\eta$ ) at a slower rate with the increase of the parameter $\zeta$ at very near the wall as well as far away of the wall. This can be explained physically as the parameter $\zeta$ increases, the fluid viscosity decreases the increment of the boundary layer thickness.

In Figure 5, variations of temperature field $\theta(\eta)$ with $\eta$ for several values of $\zeta$ (with $\operatorname{Pr}=0.71$ and $\mathrm{N}=0.1$ ) in presence of suction $(\mathrm{S}=0.5)$ are shown. It is very clear from the figure that the temperature decreases with the increasing of $\zeta$ whereas the concentration of the fluid is not significant with the increasing of $\zeta$. The increase of temperature-dependent fluid viscosity parameter $(\zeta)$ makes decrease of thermal boundary layer thickness, which results in decrease of temperature profile $\theta(\eta)$. Decrease in $\theta(\eta)$ means a decrease in the velocity of the fluid particles. So in this case the fluid particles undergo two opposite forces: one increases the fluid velocity due to decrease in the fluid viscosity (with increasing $\zeta$ ) and other decreases the fluid velocity due to decrease in temperature $\theta(\eta)$ (since $\theta(\eta)$ decreases with increasing $\zeta$ ). Near the surface, as the temperature $\theta(\eta)$ is high so the first force dominates and far away from the surface $\theta(\eta)$ is low and so the second force dominates here. Now we concentrate in the velocity and temperature distribution for the variation of suction parameter $\mathrm{S}$ in the absence and presence of temperature-dependent fluid viscosity parameter $\zeta$.

Figure 6 presents the effects of suction on fluid velocity when the fluid viscosity is uniform, i.e., $\zeta=0.5$. With the increasing value of the suction $(\mathrm{S}>0)(\zeta=$ $0.5, \operatorname{Pr}=0.71$ and $\mathrm{N}=0.1$ ), the velocity is found to decrease (Figure 6), i.e., suction causes to decrease the velocity of the fluid in the boundary layer region. The physical explanation for such a behavior is as follows. In case of suction, the heated fluid is pushed towards the wall where the buoyancy forces can act to retard the fluid due to high influence of the viscosity. This effect acts to decrease the wall shear stress. Figures $\mathbf{7}$ and $\mathbf{8}$ exhibit that the temperature $\theta(\eta)$ and concentration $\varphi(\eta)$ in boundary layer also decrease with the increasing suction parameter $\mathrm{S}(\mathrm{S}>0)(\zeta=0.5, \mathrm{Pr}=0.71$ and $\mathrm{N}=$ $0.1)$. The thermal and solutal boundary layer thickness decrease with the suction parameter $\mathrm{S}$ which causes an increase in the rate of heat and mass transfer. The explanation for such behavior is that the fluid is brought closer to the surface and reduces the thermal boundary layer thickness in case of suction. As such then the presence of wall suction decreases velocity boundary layer thicknesses but decreases the thermal and solutal boundary layers thickness, i.e., thins out the thermal and solutal boundary layers.

Figure 9 illustrates the influence of Schmidt number $S_{c}$ on the concentration. As Schmidt number $S_{c}$ increases, the mass transfer rates increases. Hence, the concentration decreases with increasing $S_{c}$. It is evident from this figure that the concentration $\varphi(\eta)$ takes its limiting value $C_{\infty}$, for higher values of the dimensionless distance $\eta$. From this figure, we observe that when the concentration difference $\Delta C$ is maintained constant, the dimensionless concentration profile decreases, in the since that the values of the Schmidt number increases. The variation in the thermal boundary layer is very small corresponding to a moderate change in Schmidt number. There are very small changes in velocity and temperature distributions when moderate changes in Schmidt number.

\section{Conclusions}

By using Lie group analysis, first find the symmetries of the partial differential equations and then reduce the equations to ordinary differential equations by using scaling and translational symmetries. Exact solutions for translation symmetry and numerical solution for scaling symmetry are obtained. From the numerical results, it is predict that the effect of increasing temperaturedependent fluid viscosity parameter on a viscous incompressible fluid is to increase the flow velocity which in 
turn, causes the temperature to decrease. It is interesting to note that the temperature of the fluid decreases at a very fast rate in the case of water in comparison with air. So, the thermal radiation effects in the presence of fluid viscosity have a substantial effect on the flow field and, thus, on the heat and mass transfer rate from the sheet to the fluid. Decrease of the concentration field due to increase in Schmidt number shows that it increases gradually as we replace Hydrogen $\left(S_{c}=0.22\right)$ by water vapour $\left(S_{c}=0.67\right)$ and Ammonia $\left(S_{c}=0.78\right)$ in the said sequence.

\section{References}

[1] L. J. Crane, "Flow Past a Stretching Plate," The Journal of Applied Mathematics and Physics, Vol. 21, No. 4, pp. 645-647.

[2] 1. H. I. Andersson, J. B. Aarseth and B. S. Dandapat, "Heat Transfer in a Liquid Film on an Unsteady Stretching Surface," International Journal of Heat and Mass Transfer, Vol. 43, No. 1, 2000, pp. 69-74.

[3] C. Y. Wang, "Liquid Film on an Unsteady Stretching Surface," Quarterly of Applied Mathematics, Vol. 48, No. 4, 1990, pp. 601-610.

[4] P. Saikrishnan and S. Roy, "Non-Uniform Slot Injection (Suction) into Steady Laminar Water Boundary Layers over (i) a Cylinder and (ii) a Sphere," International Journal of Engineering Science, Vol. 41, No. 12, 2003, pp. 1351-1365.

[5] S. Roy and P. Saikrishnan, "Non-Uniform Slot Injection (Suction) into Steady Laminar Water Boundary Layer Flow over a Rotating Sphere," International Journal of Heat and Mass Transfer, Vol. 46, No. 18, 2003, pp. 3389-3396.

[6] E. M. A. Elbashbeshy and M. A. A. Bazid, "Heat Transfer in a Porous Medium over a Stretching Surface with Internal Heat Generation and Suction or Injection," Applied Mathematics and Computation, Vol. 158, No. 3, 2004, pp. 799-807.

[7] P. Cheng and W. J. Minkowycz, "Free Convection about a Vertical Flat Plate Embedded in a Porous Medium with Application to Heat Transfer from a Disk," Journal of Geophysical Research, Vol. 82, No. 14, 1963, pp. 20402044.

[8] P. Cheng, "The Influence of Lateral Mass Flux on a Free Convection Boundary Layers in Saturated Porous Medium," International Journal of Heat and Mass Transfer, Vol. 20, No. 3, 1977, pp. 201-206.

[9] G. Wilks, "Combined Forced and Free Convection Flow on Vertical Surfaces," International Journal of Heat and Mass Transfer, Vol. 16, No. 10, 1973, pp. 1958-1964.

[10] Y. Z. Boutros, M. B. Abd-el-Malek and N. A. Badran, "Group Theoretic Approach for Solving Time-Independent Free Convective Boundary Layer Flow on a NonIsothermal Vertical Flat Plate," Archives of Mechanics, Vol. 42, No. 3, 1990, pp. 377-395.
[11] T. S. Chen and F. A. Strobel, "Buoyancy Effects in Boundary Layer Adjacent to a Continuous Moving Horizontal Flat Plate," Journal of Heat Transfer, Vol. 102, 1980, p. 170.

[12] N. Ramachandran, B. F. Armaly and T. S. Chen, "Correlation for Laminar Mixed Convection on Boundary Layers Adjacent to Inclined Continuous Moving Sheets," International Journal of Heat and Mass Transfer, Vol. 30, No. 10, 1987, pp. 2196-2199.

[13] S. L. Lee and J. S. Tsai, "Cooling of a Continuous Moving Sheet of Finite Thickness in the Presence of Natural Convection," International Journal of Heat and Mass Transfer, Vol. 33, No. 3, 1990, pp. 457-464.

[14] M. A. Hossain and H. S. Takhar, "Radiation Effect on Mixed Convection along a Vertical Plate with Uniform Surface Temperature," International Journal of Heat and Mass Transfer, Vol. 31, No. 2, 1996, pp. 243-248.

[15] H. S. Takhar, R. S. R. Gorla and V. M. Soundalgekar, "Radiation Effects on MHD Free Convection Flow of a Gas Past a Semi-Infinite Vertical Plate," The International Journal of Numerical Methods for Heat \& Fluid Flow, Vol. 6, No. 2, 1996, pp. 77-83.

[16] M. A. Hossain, M. A. Alim and D. A. Rees, "The Effect of Radiation on Free Convection from a Porous Vertical Plate," International Journal of Heat and Mass Transfer, Vol. 42, No. 7, 1999, pp. 181-191.

[17] J. Gary, D. R. Kassoy, H. Tadjeran and A. Zebib, "The Effects of Significant Viscosity Variation on Convective Heat Transport in Water-Saturated Porous Media," Journal of Fluid Mechanics, Vol. 117, 1982, pp. 233-249.

[18] K. N. Mehta and S. Sood, "Transient Free Convection Flow with Temperature-Dependent Viscosity in a Fluid Saturated Porous Medium," International Journal of Engineering Science, Vol. 30, No. 8, 1992, pp. 1083-1087.

[19] S. Mukhopadhyay, G. C. Layek and S. A. Samad, "Study of MHD Boundary Layer Flow over a Heated Stretching Sheet with Variable Viscosity," The International Journal of Numerical Methods for Heat \& Fluid Flow, Vol. 48, No. 21-22, 2005, pp. 4460-4466.

[20] S. Mukhopadhyay and G. C. Layek, "Effects of Thermal Radiation and Variable Fluid Viscosity on Free Convective Flow and Heat Transfer Past a Porous Stretching Surface," International Journal of Heat and Mass Transfer, Vol. 51, No. 2, 2008, pp. 2167-2178.

[21] M. Q. Brewster, "Thermal Radiative Transfer Properties," John Wiley and Sons, Chichester, 1972.

[22] G. K. Batchelor, "An Introduction to Fluid Dynamics," Cambridge University Press, London, 1987.

[23] J. X. Ling and A. Dybbs, "Forced Convection over a Flat Plate Submersed in a Porous Medium: Variable Viscosity Case Paper 87-WA/HT-23," American Society of Mechanical Engineers, New York, 1987.

[24] S. Gill, “A Process for the Step-by-Step Integration of Differential Equations in an Automatic Digital Computing Machine," Proceedings of the Cambridge Philosophical Society, Vol. 47, No. 1, 1951, pp. 96-108. 